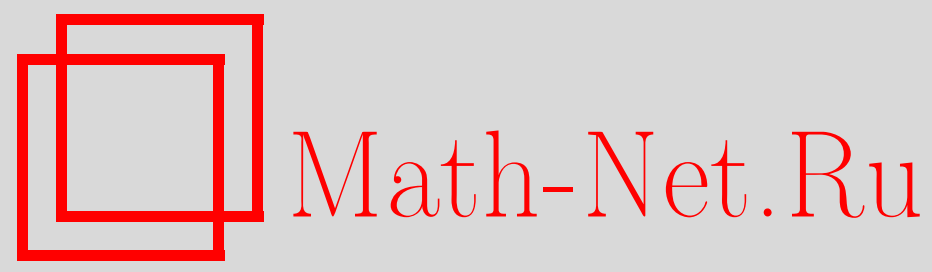

A. М. Седлецкий, О нулях функции типа МиттагЛеффлера, Матем. заметки, 2000, том 68, выпуск 5, 710724

DOI: https://doi.org/10.4213/mzm992

Использование Общероссийского математического портала Math-Net.Ru подразумевает, что вы прочитали и согласны с пользовательским соглашением http://www . mathnet.ru/rus/agreement

Параметры загрузки:

IP : 54.209 .52 .79

26 апреля 2023 г., 15:25:06

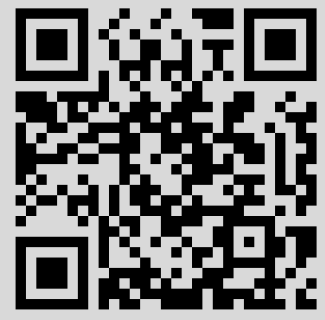


УДК 517.5

\section{О НУЛЯХ ФУНКЦИИ ТИПА МИТТАГ-ЛЕФФЛЕРА}

\section{А. М. Седлецкий}

Ранее была известна асимптотика нулей функции типа Миттаг-Леффрера

$$
E_{\rho}(z ; \mu)=\sum_{n=0}^{\infty} \frac{z^{n}}{\Gamma(\mu+n / \rho)}, \quad \rho>0, \quad \mu \in \mathbb{C},
$$

описывающая поведение нулей вне круга достаточно большого радиуса. В статье решен вопрос о числе нулей внутри такого круга, что позволило указать нумерацию всех нулей $E_{\rho}(z ; \mu)$, согласованную с асимптотикой. Рассмотрен вопрос о распределении нулей двух функций, выражающихся через $E_{1}(z ; \mu)$ : неполной гамма-функции и функции ошибок.

Библиография: 12 названий.

1. Рассматривается функиия типа Миттаг-Леффлера

$$
E_{\rho}(z ; \mu)=\sum_{n=0}^{\infty} \frac{z^{n}}{\Gamma(\mu+n / \rho)}, \quad \rho>0, \quad \mu \in \mathbb{C}
$$

(см. [1]), превращающаяся при $\mu=1$ в классическую функцию Миттаг-Леффлера $E_{\alpha}(z)=E_{1 / \alpha}(z ; 1)$. Содержательность класса целых функций $(1)$ и его замечательные асимптотические свойства объясняют интерес к вопросу о распределении нулей функции $E_{\rho}(z ; \mu)$, которьй при различных значениях $\rho$ и $\mu$ рассматривался в работах А. Вимана [2], Г. Пойа [3], М. М. Джрбашяна и А. Б. Нерсесяна (см. [1]), автора [4], И. В. Островского и И.Н. Пересёлковой [5] и других.

У функции $E_{1}(z ; \mu)=z^{1-\mu} e^{z}, \mu=1,0,-1,-2, \ldots$, нет нулей, отличных от точки $z=0$. Во всех остальных случаях функция (1) имеет бесконечное множество нулей.

Пусть $\rho>1 / 2$, причем $\mu \neq 1,0,-1,-2, \ldots$, если $\rho=1$. На множестве таких параметров введем константы

$$
\begin{gathered}
c_{\mu}= \begin{cases}\frac{1}{\rho \Gamma(\mu-1 / \rho)}, & \text { если } \mu \neq \frac{1}{\rho}-l, l \in \mathbb{Z}_{+}, \\
\frac{1}{\rho \Gamma(\mu-2 / \rho)} & \text { если } \mu=\frac{1}{\rho}-l, l \in \mathbb{Z}_{+}, \rho \neq 1,\end{cases} \\
\tau_{\mu}= \begin{cases}1+\rho(1-\mu), & \text { если } \mu \neq \frac{1}{\rho}-l, l \in \mathbb{Z}_{+}, \\
2+\rho(1-\mu), & \text { если } \mu=\frac{1}{\rho}-l, l \in \mathbb{Z}_{+}, \rho \neq 1 .\end{cases}
\end{gathered}
$$

Работа выполнена при поддержке Российского фонда фундаментальных исследований, грант № 00-01-00396. 
Кроме того, при $\rho=1 / 2, \operatorname{Re} \mu>3$ считаем, что $c_{\mu}$ и $\tau_{\mu}$ задаются первьми строками написанных формул. По построению $c_{\mu} \neq 0$ для всех рассмотренных значений $\rho$ и $\mu$. В [4] доказана следующая

Tеорема А. Пусть $\rho>0, \mu \in \mathbb{C}$, причем $\mu \neq 1,0,-1,-2, \ldots n p u \rho=1 u \operatorname{Re} \mu \neq 3$ при $\rho=1 / 2$. Тогда все достаточно большие по модулю нули $z_{n}$ функиии $E_{\rho}(z ; \mu)$ просты $u$

1) если а) $\rho>1 / 2$ или б) $\rho=1 / 2 u \operatorname{Re} \mu>3$, mo

$$
z_{n}^{\rho}=2 \pi n i-\frac{\tau_{\mu}}{\rho}\left(\log 2 \pi|n|+i \frac{\pi}{2} \operatorname{sign} n\right)+\log c_{\mu}+o(1), \quad n \rightarrow \pm \infty
$$

2) если а) $\rho<1 / 2$ или б) $\rho=1 / 2 u \operatorname{Re} \mu<3$, mo

$$
z_{n}=-\left(\frac{\pi}{\sin \pi \rho}\left(n+\frac{1}{2}+\rho(\mu-1)+o(1)\right)\right)^{1 / \rho}, \quad n \rightarrow+\infty
$$

При этом в [4] оценена скорость убьвания входящих в формулы (2), (3) бесконечно малых последовательностей $o(1)$; в частности, показано, что если $\rho=1$, то $o(1)$ есть $O((\log |n|) /|n|)$.

В формулах (2), (3) и в дальнейшем под степенной и логарифмической функциями понимаем их главные значения.

Формулы (2), (3) асимптотические; они описьвают поведение нулей функции (1) вне круга достаточно большого радиуса и не дают информации о числе нулей этой функции внутри указанного круга. Очевидно, вопрос о числе таких нулей равносилен вопросу о согласовании асимптотики с нумерацией нулей.

ОПРЕДЕЛЕНИЕ. Пусть $Z=\left(z_{n}\right)$ - множество всех нулей целой функции $F(z)$, и пусть имеет место асимптотическая формула

$$
\left.z_{n}=\varphi(n)+o(1), \quad n \rightarrow \pm \infty \quad \text { или } n \rightarrow+\infty\right),
$$

где $\varphi(n) \rightarrow \infty$. Скажем, что асимптотика (4) согласована с нумерацией нулей функции $F(z)$ при помощи множества индексов $T$, если существует биекция $T \leftrightarrow Z$, сохраняющая асимптотику (4).

При этом мы должны считать, что корню $\lambda$ кратности $m$ в $Z$ отвечают элементы $z_{s+1}=\cdots=z_{s+m}=\lambda$ с различными номерами.

Поясним определение на примерах функций $\sin z$ и $z^{-1} \sin z$, нули которых имеют одинаковую асимптотику $z_{n}=\pi n+o(1), n \rightarrow \pm \infty$. Но в первом случае она согласована с нумерацией нулей при помощи множества индексов $\mathbb{Z}$, а во втором - при помощи множества $\mathbb{Z} \backslash\{0\}$.

В настоящей статье мы находим множества индексов $T$, при помощи которых асимптотические формулы (2), (3) согласованы с нумерацией нулей функции (1). Имеют место следуюшие утверждения. 
Teорема 1. 1) Ecлu a) $\rho>1 / 2 u \mu \neq 1 / \rho-l, l \in \mathbb{Z}_{+}, u л u$ б) $\rho=1 / 2 u \operatorname{Re} \mu>3, m o$ асимптотика (2) согласована с нумерачией нулей функции $E_{\rho}(z ; \mu)$ при помощи множества индексов $\mathbb{Z} \backslash\{0\}$.

2) Если $\rho>1 / 2, \rho \neq 1$ и $\mu=1 / \rho-l, l \in \mathbb{Z}_{+}$, то асимптотика (2) согласована $c$ нумерацией нулей функиии $E_{\rho}(z ; \mu)$ при помощи множества индексов $\mathbb{Z} \backslash\{0 ; 1\}$.

Tеорема 2. Если а) $\rho<1 / 2$ или б) $\rho=1 / 2 u \operatorname{Re} \mu<3$, то асимптотика (3) согласована с нумерачией нулей функиии $E_{\rho}(z ; \mu)$ при помощи множества индексов $\mathbb{Z}_{+}$.

Для иллюстрации рассмотрим функции

$$
E_{1}(z ; 2)=\frac{e^{z}-1}{z}, \quad E_{1 / 2}(z ; 1)=\operatorname{ch} \sqrt{z} .
$$

$\mathrm{B}$ первом случае $\tau_{\mu}=0, c_{\mu}=1$ и по теореме $\mathrm{A}$ асимптотика нулей $z_{n}$ этих функций имеет соответственно вид

$$
z_{n}=2 \pi n i+o(1), \quad n \rightarrow \pm \infty, \quad z_{n}=-\pi^{2}\left(n+\frac{1}{2}+o(1)\right)^{2}, \quad n \rightarrow+\infty .
$$

Теоремы 1, 2 утверждают, что эти асимптотики согласованы с нумерацией нулей при помощи множеств индексов $\mathbb{Z} \backslash\{0\}$ и $\mathbb{Z}_{+}$соответственно. С другой стороны, в силу явного задания функций мы имеем точные формулы $z_{n}=2 \pi n i, n \in \mathbb{Z} \backslash\{0\}$, и $z_{n}=$ $-\pi^{2}(n+1 / 2)^{2}, n \in \mathbb{Z}_{+}$.

Кроме того, мы болееподробно остановимся на распределении нулей функции $E_{1}(z ; \mu)$ и двух выражающихся через нее функций, а именно неполной гамма-функции и функции ошибок.

\section{2. Оценка интеграла от логарифма модуля.}

2а. Случай $\rho>1 / 2$. Пусть $\alpha \in(\pi /(2 \rho), \min (\pi / \rho, \pi))$ и $s \in \mathbb{N}$. Тогда верны асимптотические оценки $(r=|z|)$ :

$$
\begin{aligned}
E_{\rho}(z ; \mu) & =\rho z^{\rho(1-\mu)} \exp \left(z^{\rho}\right)-\sum_{k=1}^{s} \frac{z^{-k}}{\Gamma(\mu-k / \rho)}+O\left(\frac{1}{r^{s+1}}\right), \quad|\arg z| \leqslant \alpha, \\
-E_{\rho}(z ; \mu) & =-\sum_{k=1}^{s} \frac{z^{-k}}{\Gamma(\mu-k / \rho)}+O\left(\frac{1}{r^{s+1}}\right), \quad \alpha \leqslant|\arg z| \leqslant \pi,
\end{aligned}
$$

равномерные в соответствуюших секторах $[1$, с. 134]. Введем множества

$$
\begin{aligned}
P_{-} & =\left(z: r>1, \operatorname{Re}\left(z^{\rho}+\tau_{\mu} \log z\right)-\log \left|c_{\mu}\right| \leqslant-1\right), \\
P_{+} & =\left(z: r>1, \operatorname{Re}\left(z^{\rho}+\tau_{\mu} \log z\right)-\log \left|c_{\mu}\right| \geqslant 1\right), \\
P & =\left(z: r>1,-1<\operatorname{Re}\left(z^{\rho}+\tau_{\mu} \log z\right)-\log \left|c_{\mu}\right|<1\right) .
\end{aligned}
$$

$\Phi$ иксируем $\alpha$ в указанном выше интервале и полагаем $s=1$ при $\mu \neq 1 / \rho-l, l \in \mathbb{Z}_{+}$, и $s=2$ при $\mu=1 / \rho-l, l \in \mathbb{Z}_{+}$. Как отмечалось, $c_{\mu} \neq 0$. 
В силу формулы (6)

$$
z^{s} E_{\rho}(z ; \mu) \rightarrow-\rho c_{\mu} \neq 0, \quad r \rightarrow \infty, \quad \alpha \leqslant|\arg z| \leqslant \pi .
$$

Пусть $|\arg z| \leqslant \alpha$ и $z \in P_{-}$. Тогда из определения множества $P_{-}$и из формулы (5) следует, что

$$
\begin{aligned}
& \left|\frac{1}{\rho} z^{s} E_{\rho}(z ; \mu)\right| \leqslant \frac{\left|c_{\mu}\right|}{e}+\left|c_{\mu}\right|+O\left(\frac{1}{r}\right) \leqslant M<\infty, \quad r>r_{0}, \\
& \left|\frac{1}{\rho} z^{s} E_{\rho}(z ; \mu)\right| \geqslant\left|c_{\mu}\right|-\frac{\left|c_{\mu}\right|}{e}+O\left(\frac{1}{r}\right) \geqslant m>0, \quad r>r_{0} .
\end{aligned}
$$

Объединяя (7) с (8) и (9), получаем

$$
\left|z^{s} E_{\rho}(z ; \mu)\right| \asymp 1, \quad r>r_{0}, \quad z \in P_{-} .
$$

Пусть $z \in P_{+}$. Тогда по формуле (5)

$$
\begin{aligned}
& \exp \left(\operatorname{Re}\left(z^{\rho}+\tau_{\mu} \log z\right)\right)-\left|c_{\mu}\right|+O\left(\frac{1}{r}\right) \\
& \leqslant\left|\frac{1}{\rho} z^{s} E_{\rho}(z ; \mu)\right| \leqslant \exp \left(\operatorname{Re}\left(z^{\rho}+\tau_{\mu} \log z\right)\right)+\left|c_{\mu}\right|+O\left(\frac{1}{r}\right) .
\end{aligned}
$$

Но по определению множества $P_{+}$

$$
\left|c_{\mu}\right| \leqslant e^{-1} \cdot \exp \left(\operatorname{Re}\left(z^{\rho}+\tau_{\mu} \log z\right)\right), \quad z \in P_{+} .
$$

Подставляя (12) в (11), получаем оценку

$$
\left|z^{s} E_{\rho}(z ; \mu)\right| \asymp \exp \left(\operatorname{Re}\left(z^{\rho}+\tau_{\mu} \log z\right)\right), \quad r>r_{0}, \quad z \in P_{+} .
$$

Осталось рассмотреть "криволинейную полосу" $P$. При отображении

$$
w=z^{\rho}+\tau_{\mu} \log z
$$

она перейдет в вертикальную полосу $\gamma_{-}<\operatorname{Re} w<\gamma_{+}, \gamma_{ \pm}=\log \left|c_{\mu}\right| \pm 1$, из которой удалено некоторое ограниченное множество. В силу теоремы Руше при достаточно больших $|z|$ отображение (14) однолистно. Рассмотрим образ $e(w)$ функции $(1 / \rho) z^{s} E_{\rho}(z ; \mu)$ при этом отображении. В силу формулы (5) при $\gamma_{-}<\operatorname{Re} w<\gamma_{+},|\operatorname{Im} w| \rightarrow \infty$

$$
e(w)=e^{w}-c_{\mu}+o(1)=: \varphi(w)+o(1) .
$$

Функция $\varphi(w)$ периодична с периодом $2 \pi i$. Отсюда и из ее непрерьвности следует, что $0<c(\delta) \leqslant|\varphi(w)| \leqslant c$ при $\gamma_{-}<\operatorname{Re} w<\gamma_{+},|\operatorname{Im} w|>r_{0}$ и вне кружков радиуса $\delta \mathrm{c}$ центрами в нулях $\varphi(w)$. В силу $(15)$

$$
0<m \leqslant|e(w)| \leqslant M<\infty, \quad \gamma_{-}<\operatorname{Re} w<\gamma_{+}, \quad|\operatorname{Im} w|>r_{0}
$$


вне кружков радиуса $\delta$ с центрами в нулях $w_{n}$ функции $e(w)$, имеющих вид $w_{n}=2 \pi n i+$ $\log c_{\mu}+o(1)$. Беря $\delta$ достаточно малым и возвращаясь к переменной $z$, из (16) делаем вывод, что найдутся окружности $|z|=r_{k} \rightarrow \infty$ такие, что на их пересечении с $P$ верна оценка (10), т.е.

$$
\left|z^{s} E_{\rho}(z ; \mu)\right| \asymp 1, \quad z \in P, \quad|z|=r_{k} \rightarrow \infty .
$$

Оценки $(10),(13),(17)$ показьвают, что на подходящих окружностях справедливы оценки

$$
\begin{gathered}
\log \left|E_{\rho}(z ; \mu)\right|=\operatorname{Re} z^{\rho}+\left(\operatorname{Re} \tau_{\mu}-s\right) \log r+O(1), \quad z \in P_{+}, \quad|z|=r_{k} \rightarrow \infty \\
\log \left|E_{\rho}(z ; \mu)\right|=-s \log r+O(1), \quad z \in P_{-} \cup P, \quad|z|=r_{k} \rightarrow \infty
\end{gathered}
$$

С помощью этих оценок выясним поведение интеграла

$$
I(r)=\frac{1}{2 \pi} \int_{-\pi}^{\pi} \log \mid E_{\rho}\left(r e^{i \theta} ; \mu\right) d \theta, \quad r=r_{k} \rightarrow \infty .
$$

Обозначим через $\alpha_{ \pm}(r)$ аргументы точек, которые ограничивают дугу окружности $|z|=r$, попавшую на множество $P_{+}, \alpha_{-}(r)<0<\alpha_{+}(r)$. Это означает, что точки $z=r \exp \left(i \alpha_{ \pm}(r)\right)$ удовлетворяют условию

$$
\operatorname{Re}\left(z^{\rho}+\tau_{\mu} \log z\right)-\log \left|c_{\mu}\right|=1, \quad|z|>1,
$$

из которого находим, что обе величины $\pm \alpha_{ \pm}(r)$ имеют вид

$$
\frac{\pi}{2 \rho}+O\left(\frac{\log r}{r^{\rho}}\right)=: \alpha(r)
$$

Интеграл $I(r)$ запишем в виде суммы интегралов $I_{j}, j=1,2,3,4$, которые берутся соответственно по интервалам $\left(0, \alpha_{+}(r)\right),\left(\alpha_{-}(r), 0\right),\left(\alpha_{+}(r), \pi\right),\left(-\pi, \alpha_{-}(r)\right)$. Для оценки $I_{1}, I_{2}$ применяем формулу (18), а для оценки $I_{3}, I_{4}-$ формулу (19). С учетом (20) имеем $I_{3}=\frac{1}{2 \pi} \int_{\alpha(r)}^{\pi}(O(1)-s \log r) d \theta=O(1)-\frac{s}{2 \pi}(\pi-\alpha(r)) \log r=s\left(\frac{1}{4 \rho}-\frac{1}{2}\right) \log r+O(1)$.

Аналогичная оценка верна для $I_{4}$. Далее, так как в силу (20)

$$
\sin \rho \alpha(r)=\cos \left(O\left(\frac{\log r}{r^{\rho}}\right)\right)=1+O\left(\frac{\log ^{2} r}{r^{2 \rho}}\right)
$$

TO

$$
\begin{aligned}
I_{1} & =\frac{1}{2 \pi} \int_{0}^{\alpha(r)}\left(r^{\rho} \cos \rho \theta+\left(\operatorname{Re} \tau_{\mu}-s\right) \log r+O(1)\right) d \theta \\
& =\frac{r^{\rho}}{2 \pi \rho} \sin \rho \alpha(r)+\frac{\alpha(r)}{2 \pi}\left(\operatorname{Re} \tau_{\mu}-s\right) \log r+O(1) \\
& =\frac{r^{\rho}}{2 \pi \rho}+\frac{1}{4 \rho}\left(\operatorname{Re} \tau_{\mu}-s\right) \log r+O(1)
\end{aligned}
$$


и аналогичная оценка верна для $I_{2}$. Объединяя оценки для $I_{j}$, получаем промежуточный итог:

$$
\frac{1}{2 \pi} \int_{-\pi}^{\pi} \log \left|E_{\rho}\left(r e^{i \theta} ; \mu\right)\right| d \theta=\frac{r^{\rho}}{\pi \rho}+\left(\frac{\operatorname{Re} \tau_{\mu}}{2 \rho}-s\right) \log r+O(1), \quad r=r_{k} \rightarrow \infty .
$$

2б. Случай $\rho=1 / 2, \operatorname{Re} \mu>3$. Справедливы представления

$$
E_{1 / 2}(z ; \mu)=\frac{1}{2} z^{(1-\mu) / 2}\left(e^{\sqrt{z}}+e^{\mp i(1-\mu) \pi} \cdot e^{-\sqrt{z}}\right)-\frac{z^{-1}}{\Gamma(\mu-2)}+O\left(\frac{1}{r^{2}}\right)
$$

соответственно при $0 \leqslant \arg z \leqslant \pi$ и $-\pi \leqslant \arg z \leqslant 0$ (см. [1, с. 136]). Напомним, что в рассматриваемом случае константы $c_{\mu}$ и $\tau_{\mu}=\tau$ вычисляются по первьм строкам задающих их формул (см. п. 1). Имеем $c_{\mu} \neq 0, \operatorname{Re} \tau<0$. Пусть $0 \leqslant \arg z \leqslant \pi$. Тогда

$$
\begin{aligned}
2 z E_{1 / 2}(z ; \mu) & =\exp (\sqrt{z}+\tau \log z)+e^{-i \pi(1-\mu)} z^{\tau} e^{-\sqrt{z}}-c_{\mu}+O\left(\frac{1}{r}\right) \\
& =\exp (\sqrt{z}+\tau \log z)-c_{\mu}+o(1), \quad r \rightarrow \infty
\end{aligned}
$$

так как $\operatorname{Re} \tau<0$. Введем множества

$$
\begin{aligned}
P_{+} & =\left(z: r>1, \operatorname{Re}(\sqrt{z}+\tau \log z)-\log \left|c_{\mu}\right| \geqslant 1\right), \\
P & =\left(z: r>1, \operatorname{Re}(\sqrt{z}+\tau \log z)-\log \left|c_{\mu}\right|<1\right) .
\end{aligned}
$$

Пусть $0 \leqslant \arg z \leqslant \pi, z \in P_{+}$. Тогда имеет место неравенство (12) с $\rho=1 / 2$. Поэтому из (22) вытекает оценка (13) с $\rho=1 / 2, s=1$.

Рассмотрим отображение (14) с $\rho=1 / 2$. При этом отображении образ области $P \cap(\operatorname{Im} z>0)$ для достаточно больших $\operatorname{Im} w$ совпадает с расширяющейся криволинейной полуполосой (обозначим ее через $V$ ), которая ограничена: справа вертикальной прямой $\operatorname{Re} w=1+\log \left|c_{\mu}\right|:=b$, а слева кривой $w=i\left(r^{1 / 2}+(\operatorname{Im} \tau) \log r\right)+(\operatorname{Re} \tau) \log r+c$, $r>r_{0}$. Из $(22)$ следует, что образ $e(w)$ функции $2 z E_{1 / 2}(z ; \mu)$ при рассматриваемом отображении имеет вид (15). Фиксируем $a<0$ так, чтобы $\left|e^{w}\right|=\exp (\operatorname{Re} w) \leqslant\left|c_{\mu}\right| / 2$ при $\operatorname{Re} w<a$. Введем полуполосу $V_{+}=\left(w: a<\operatorname{Re} w<b, \operatorname{Im} w>r_{0}\right)$ и пусть $V_{-}=V \backslash V_{+}$. В силу выбора $a$ из (15) следует, что

$$
|e(w)| \geqslant \frac{\left|c_{\mu}\right|}{2}+o(1) \geqslant m>0, \quad w \in V_{-}, \quad \operatorname{Im} w>r_{0}
$$

Если же $w \in V_{+}$, то, используя периодичность $e^{w}$, заключаем, что оценка снизу $|e(w)| \geqslant$ $m>0$ верна и для точек $w \in V_{+}$, лежащих вне кружков $K_{n}(\delta)$ радиуса $\delta$ с центрами в корнях функции $e(w)$. Итак,

$$
|e(w)| \geqslant c(\delta)>0, \quad w \in V, \quad w \notin \cup K_{n}(\delta) .
$$

Оценка сверху $|e(w)| \leqslant M<\infty$ для $w \in V$ сразу следует из (15) и из определения множества $V$. Значит, $|e(w)| \asymp 1, w \in V, w \notin \cup K_{n}(\delta)$. Беря $\delta$ достаточно мальм 
и возвращаясь на плоскость $z$, заключаем, что найдется последовательность $r_{k} \rightarrow \infty$ такая, что вьполняется оценка (17) с $\rho=1 / 2, s=1$.

Оценки (13) и (17) (с $\rho=1 / 2, s=1)$ установлены нами для $0 \leqslant \arg z \leqslant \pi$. Аналогично они доказываются и для $-\pi \leqslant \arg z \leqslant 0$. Значит, эти оценки с $s=1$ верны для случая $\rho=1 / 2, \operatorname{Re} \mu>3$ без ограничений на $\arg z$. В итоге для случая $\rho=1 / 2, \operatorname{Re} \mu>3$ мы приходим к оценкам (18) и (19) с $s=1$, причем в (19) множество $P_{-}$теперь отсутствует. После чего повторяются рассуждения, следующие за (19); при этом достаточно сделать формальную замену $\rho$ на $1 / 2$ и $s$ на 1 . В результате получаем оценку типа $(21)$ :

$$
\frac{1}{2 \pi} \int_{-\pi}^{\pi} \log \left|E_{\rho}\left(r e^{i \theta} ; \mu\right)\right| d \theta=\frac{2}{\pi} r^{1 / 2}+\left(\operatorname{Re} \tau_{\mu}-1\right) \log r+O(1), \quad r=r_{k} \rightarrow \infty .
$$

2в. Случаи $\rho=1 / 2, \operatorname{Re} \mu<3$ и $\rho<1 / 2$. На этот раз применяем формулу

$$
E_{\rho}(z ; \mu)=\rho z^{\rho(1-\mu)} \sum_{|\arg z+2 \pi n| \leqslant \pi /(2 \rho)+\varepsilon} e^{2 \pi n i \rho(1-\mu)} \cdot \exp \left(z^{\rho} e^{2 \pi n i \rho}\right)+O\left(\frac{1}{r}\right),
$$

справедливую при всех достаточно малых $\varepsilon>0$. Эта формула с $\varepsilon=0$ доказана в $[1$, c. 137], но с $\varepsilon=0$ она, вообще говоря, не является равномерной. Желаемая равномерность как раз и достигается присутствием $\varepsilon>0$; ведь формула (24) вьводится из формулы (5), где значение $\alpha$ превосходит значение $\pi /(2 \rho)$. Понадобятся и уточняющие результат А. $\Phi$. Леонтьева [6], [7] факты о поведении квазиполинома

$$
R(w)=\sum_{j=1}^{m} h_{j} e^{\gamma_{j} w}, \quad h_{j} \neq 0
$$

где $\gamma_{j}$ - вершины вьпуклого многоугольника $G \supset 0, \arg \gamma_{j}<\arg \gamma_{j+1}$. Обозначим через $\kappa(\varphi)$ опорную функцию $G$, т.е.

$$
\kappa(\varphi)=\sup \left(\operatorname{Re}\left(w e^{-i \varphi}\right): w \in G\right)
$$

Лемма 1 [8]. 1) Последовательность $\Lambda$ нулей квазиполинома (25) имеет вид $\Lambda=\Lambda_{1} \cup \cdots \cup \Lambda_{m}$, где подпоследовательность $\Lambda_{j}$ асимптотически распределена вдоль луча $\arg w=\pi / 2-\arg \left(\gamma_{j+1}-\gamma_{j}\right)$ по закону арифметической прогрессии, $j=1, \ldots, m$.

2) Вне кружкков одинакового радиуса с иентрами в нулях $R(w)$

$$
|R(w)| \asymp \exp (\kappa(\arg w)|w|) .
$$

Пусть $|\arg z| \leqslant \pi$. Тогда в сумме (24) обязательно присутствует индекс $n=0$. Полагая $w=z^{\rho},|\arg w| \leqslant \pi \rho$ и фиксируя достаточно малое $\varepsilon>0$, запишем эту сумму в виде

$$
Q(w)=\sum_{|\arg w+2 \pi n \rho| \leqslant \pi / 2+\varepsilon} h_{n} \exp \left(w e^{2 \pi n \rho i}\right), \quad h_{n}=e^{2 \pi n i \rho(1-\mu)} .
$$

В соответствии с суммированием в $(26)$ сектор $|\arg w| \leqslant \pi \rho$ разбивается на конечное число секторов $S_{j}$ с общей вершиной в точке 0 без общих внутренних точек, причем 
каждому сектору $S_{j}$ в $(26)$ отвечает свое множество индексов $I_{j}$. При любом $j$ точки $\exp (2 \pi n \rho i), n \in I_{j}$, являются вершинами некоторого выпуклого многоугольника $G_{j}$ (которьй может вырождаться в отрезок или в точку $w_{0}=1$ ), одной из вершин которого служит точка 1 . Пусть $Q_{j}(w)$ - соответствующий ему квазиполином, т.е. часть суммы в $(26)$, отвечающая индексам $n \in I_{j}$. Пусть $\kappa_{j}(\varphi)$ - опорная функция $G_{j}$. Тогда по лемме 1

$$
\left|Q_{j}(w)\right| \asymp \exp \left(\kappa_{j}(\arg w)|w|\right), \quad w \in S_{j}, \quad w \notin D_{j}(\delta),
$$

где $D_{j}(\delta)$ есть объединение кружков радиуса $\delta$ с центрами в нулях функции $Q_{j}(w)$. Но $Q_{j}(w)=Q(w)$ в секторе $S_{j}$. Далее, точка $w_{0}=1$ является одной из вершин многоугольника $G_{j}$, поэтому его опорная функция $\kappa_{j}(\varphi)$ в секторе $|\arg w| \leqslant \pi \rho$ совпадает с опорной функцией точки $w_{0}=1$, т.е. $\kappa_{j}(\varphi)=\cos \varphi,|\varphi| \leqslant \pi \rho$, при всех $j$. Так как набор чисел $j$ конечен, то из $(27)$ следует, что вне $\cup D_{j}(\delta)$

$$
|Q(w)| \asymp \exp (|w| \cos \varphi), \quad|\varphi|=|\arg w| \leqslant \pi \rho .
$$

По лемме 1 в сектор $|\arg w| \leqslant \pi \rho$ могут попасть части только тех кружков из $D_{j}(\delta)$, центры которых асимптотически распределены вдоль лучей $\arg w= \pm \pi \rho$ по закону арифметической прогрессии. Отсюда и из конечности набора чисел $j$ следует, что, взяв $\delta$ достаточно мальм, мы можем быть уверены в оценке (28) на частях подходящих окружностей $|w|=R_{k} \rightarrow \infty$, попавших в сектор $|\arg w| \leqslant \pi \rho$. Значит, если $|\theta|=|\arg z| \leqslant \pi$, то

$$
\left|Q\left(z^{\rho}\right)\right| \asymp \exp \left(|z|^{\rho} \cos \rho \theta\right), \quad|z|=r_{k}=R_{k}^{1 / \rho} \rightarrow \infty .
$$

Запишем формулу (24) в виде

$$
z^{\rho(\mu-1)} E_{\rho}(z ; \mu)=\rho Q\left(z^{\rho}\right)+O\left(\frac{1}{r^{1+\rho(1-\operatorname{Re} \mu)}}\right) .
$$

Если $\rho<1 / 2$, то $\cos \rho \theta \geqslant \cos \pi \rho>0$ и из (29) следует, что в правой части (30) доминирует первое слагаемое. Если $\rho=1 / 2$, то $\cos \rho \theta \geqslant 0$ и $\left|Q\left(z^{\rho}\right)\right| \geqslant m>0$. Но $\operatorname{Re} \mu<3$ и потому $O\left(1 / r^{1+\rho(1-\operatorname{Re} \mu)}\right)$ есть $o(1)$ при $r \rightarrow \infty$; значит, и в случае $\rho=1 / 2, \operatorname{Re} \mu<3$ первое слагаемое в правой части (30) является доминирующим. В итоге

$$
\left|E_{\rho}(z ; \mu)\right| \asymp r^{\rho(1-\operatorname{Re} \mu)} \exp \left(r^{\rho} \cos \rho \theta\right), \quad|z|=r=r_{k} \rightarrow \infty .
$$

Отсюда

$$
\frac{1}{2 \pi} \int_{-\pi}^{\pi} \log \left|E_{\rho}\left(r e^{i \theta} ; \mu\right)\right| d \theta=\frac{r^{\rho}}{\pi \rho} \sin \pi \rho+\rho(1-\operatorname{Re} \mu) \log r+O(1), \quad r=r_{k} \rightarrow \infty
$$

\section{3. Оценка усредненной плотности положительной последовательности.}

ЛЕмма 2. Обозначим через $n(t)$ число точек положительной последовательности $\left(z_{n}\right)_{n=m}^{+\infty}$ в интервале $(0, t), t>0$, и пусть

$$
z_{n}=(a n+b \log n+d+o(1))^{1 / \rho}, \quad n \rightarrow+\infty,
$$

где $a, \rho>0, b, d \in \mathbb{R}$. Тогда при $r \rightarrow+\infty$

$$
N(r)=\int_{0}^{r} \frac{n(t)}{t} d t=\frac{r^{\rho}}{a \rho}-\frac{b \rho}{2 a} \log ^{2} r+\left(\frac{1}{2}-m-\frac{d}{a}+\frac{b}{a} \log a\right) \log r+o(\log r) .
$$


ДокАЗАТЕЛЬСтво. Фиксируем $\varepsilon>0$. Введем последовательности $x_{n}^{+}$и $x_{n}^{-}$формулами

$$
x_{n}^{ \pm}=a n+b \log n+d \pm \varepsilon, \quad n=k, k+1, \ldots,
$$

где $k \in \mathbb{N}$ выбрано столь большим, чтобы входящая в (32) величина $o(1)$ удовлетворяла неравенству $|o(1)|<\varepsilon, n \geqslant k$, и чтобы последовательность $x_{n}^{-}$была положительной и возрастающей. Пусть $n_{ \pm}(t)$ - число точек $x_{n}^{ \pm}$на интервале $(0, t)$. Так как число точек $z_{n}$ на интервале $(0, t)$ равно числу точек $z_{n}^{\rho}$ на интервале $\left(0, t^{\rho}\right)$, то

$$
k-m+n_{+}\left(t^{\rho}\right) \leqslant n(t) \leqslant k-m+n_{-}\left(t^{\rho}\right), \quad t>x_{k}^{+},
$$

и, значит, при всех достаточно больших $r$

$$
M_{1}+(k-m) \log r+\int_{x_{k}^{+}}^{r} \frac{n_{+}\left(t^{\rho}\right)}{t} d t \leqslant N(r) \leqslant M_{2}+(k-m) \log r+\int_{x_{k}^{-}}^{r} \frac{n_{-}\left(t^{\rho}\right)}{t} d t .
$$

Далее, так как $n_{ \pm}(t)=n-k+1, t \in\left(x_{n}^{ \pm}, x_{n+1}^{ \pm}\right)$, то при $N:=\max \left(n:\left(x_{n}^{-}\right)^{1 / \rho} \leqslant r\right)$ имеем

$$
\begin{aligned}
& \rho \int_{x_{k}^{ \pm}}^{r} \frac{n_{ \pm}\left(t^{\rho}\right)}{t} d t=\rho \sum_{n=k}^{N}(n-k+1) \int_{\left(x_{n}^{ \pm}\right)^{1 / \rho}}^{\left(x_{n+1}^{ \pm}\right)^{1 / \rho}} \frac{d t}{t}+O(1) \\
& \quad=\sum_{n=k}^{N}(n-k+1) \log \frac{x_{n+1}^{ \pm}}{x_{n}^{ \pm}}+O(1) \\
& \quad=\sum_{n=k}^{N}(n-k+1) \log \left(1+\frac{a+b \log (1+1 / n)}{a n+b \log n+d \pm \varepsilon}\right)+O(1) \\
& =\sum_{n=k}^{N}(n-k+1)\left(\frac{a+b \log (1+1 / n)}{a n+b \log n+d \pm \varepsilon}-\frac{1}{2}\left(\frac{a+b \log (1+1 / n)}{a n+b \log n+d \pm \varepsilon}\right)^{2}\right)+O(1) .
\end{aligned}
$$

$\mathrm{B}$ правой части заменим $\log (1+1 / n)$ в первом слагаемом на $1 / n$, а во втором на 0 ; остаточньй член формулы Тейлора для $\log (1+1 / n)$ даст при суммировании ограниченную по $N$ величину. Получим

$$
\begin{aligned}
& \rho \int_{x_{k}^{ \pm}}^{r} \frac{n_{ \pm}\left(t^{\rho}\right)}{t} d t=\sum_{n=k}^{N}\left(\frac{a n-a(k-1)+b}{a n+b \log n+d \pm \varepsilon}-\frac{1}{2} \frac{a^{2} n}{(a n+b \log n+d \pm \varepsilon)^{2}}\right)+O(1) \\
& \quad=\sum_{n=k}^{N}\left(1-\frac{b \log n}{a n+b \log n+d \pm \varepsilon}+\frac{b-a(k-1)-d \mp \varepsilon}{a n+b \log n+d \pm \varepsilon}\right)-\frac{1}{2} \log N+O(1) \\
& \quad=N-\frac{b}{2 a} \log ^{2} N+\left(\frac{b-a(k-1)-d \mp \varepsilon}{a}-\frac{1}{2}\right) \log N+O(1) .
\end{aligned}
$$

Теперь выразим $N$ через $r$ из условия: $N$ есть наибольший из индексов $n$, для которых $a n+b \log n+d-\varepsilon \leqslant r^{\rho}$. Очевидно,

$$
N=\frac{r^{\rho}}{a}-\frac{b \rho}{a} \log r+O(1)
$$


и, значит, $\log N=\rho \log r-\log a+o(1), \log ^{2} N=\rho^{2} \log ^{2} r-2 \rho \log a \log r+o(\log r)$. Подставляя эти выражения для $N, \log N, \log ^{2} N$ в (34), а затем полученное - в (33), видим, что интеграл $N(r)$ заключен между величинами

$$
\frac{r^{\rho}}{a \rho}-\frac{\rho b}{2 a} \log ^{2} r+\left(\frac{1}{2}-m+\frac{ \pm \varepsilon-d}{a}+\frac{b}{a} \log a\right) \log r+o(\log r), \quad r \rightarrow \infty .
$$

Так как $\varepsilon$ можно брать произвольно малым, то отсюда следует требуемая асимптотика. Лемма 2 доказана.

4. Доказательство теорем 1, 2. Если точка $z=0$ является корнем функции $E_{\rho}(z ; \mu)$ кратности $m$, то перейдем к функции

$$
F(z)=\left(\frac{z-\lambda}{z}\right)^{m} \cdot E_{\rho}(z ; \mu), \quad F(0) \neq 0,
$$

где $E_{\rho}(\lambda ; \mu) \neq 0$. Полагаем $F(z)=E_{\rho}(z ; \mu)$, если $E_{\rho}(0 ; \mu) \neq 0$. Теоремы 1,2 достаточно доказать для функции $F(z)$.

Пусть мы находимся в условиях теоремы 1 . По теореме А последовательность $Z$ корней $z_{n}$ функции $F(z)$ представима в виде

$$
Z=Z_{+} \cup Z_{-}, \quad Z_{+} \cap Z_{-}=\varnothing, \quad Z_{+}=\left(z_{n}\right)_{n=m}^{+\infty}, \quad Z_{-}=\left(z_{n}\right)_{n=-1}^{-\infty},
$$

где $m$ - некоторое целое число. При этом если $|n|$ достаточно велик, то $\operatorname{Im} z_{n} \gtrless 0$ соответственно для $z_{n} \in Z_{ \pm}$. Обе последовательности $\left|Z_{+}\right|=\left(\left|z_{n}\right|\right)_{m}^{\infty},\left|Z_{-}\right|=\left(\left|z_{n}\right|\right)_{-1}^{-\infty}$ положительны.

В силу (2)

$$
\begin{aligned}
\operatorname{Im} z_{n}^{\rho} & =2 \pi n-\frac{1}{\rho}\left(\operatorname{Im} \tau_{\mu}\right) \log 2 \pi|n|-\frac{1}{\rho}\left(\operatorname{Re} \tau_{\mu}\right) \frac{\pi}{2} \operatorname{sign} n+\arg c_{\mu}+o(1), \\
\operatorname{Re} z_{n}^{\rho} & =a_{1} \log 2 \pi|n|+a_{2} \operatorname{sign} n+a_{3}+o(1),
\end{aligned}
$$

где $a_{j}$ - некоторые константы. Поэтому

$$
\begin{aligned}
\left|z_{n}\right|^{\rho} & =\sqrt{\left(\operatorname{Im} z_{n}^{\rho}\right)^{2}+\left(\operatorname{Re} z_{n}^{\rho}\right)^{2}}=\left|\operatorname{Im} z_{n}^{\rho}\right| \sqrt{1+\frac{\left(\operatorname{Re} z_{n}^{\rho}\right)^{2}}{\left(\operatorname{Im} z_{n}^{\rho}\right)^{2}}} \\
& =\left|\operatorname{Im} z_{n}^{\rho}\right|\left(1+O\left(\frac{\log ^{2}|n|}{n^{2}}\right)\right)=\left|\operatorname{Im} z_{n}^{\rho}\right|+o(1)
\end{aligned}
$$

и, значит,

$$
\left|z_{n}\right|^{\rho}=\left|2 \pi n-\frac{1}{\rho}\left(\operatorname{Im} \tau_{\mu}\right) \log \right| n\left|+\arg c_{\mu}-\frac{\pi}{2 \rho}\left(\operatorname{Re} \tau_{\mu}\right) \operatorname{sign} n-\frac{1}{\rho}\left(\operatorname{Im} \tau_{\mu}\right) \log 2 \pi+o(1)\right| .
$$

Таким образом, если $z_{n} \in Z_{+}$, то

$$
\left|z_{n}\right|=\left(2 \pi n-\frac{1}{\rho}\left(\operatorname{Im} \tau_{\mu}\right) \log n+\arg c_{\mu}-\frac{\pi}{2 \rho} \operatorname{Re} \tau_{\mu}-\frac{1}{\rho}\left(\operatorname{Im} \tau_{\mu}\right) \log 2 \pi+o(1)\right)^{1 / \rho}, \quad n \rightarrow+\infty
$$


и если $z_{n} \in Z_{-}$, то

$\left|z_{-n}\right|=\left(2 \pi n+\frac{1}{\rho}\left(\operatorname{Im} \tau_{\mu}\right) \log n-\arg c_{\mu}-\frac{\pi}{2 \rho} \operatorname{Re} \tau_{\mu}+\frac{1}{\rho}\left(\operatorname{Im} \tau_{\mu}\right) \log 2 \pi+o(1)\right)^{1 / \rho}, \quad n \rightarrow+\infty$.

Следовательно, для последовательностей $\left|Z_{+}\right|$и $\left|Z_{-}\right|$вьполнены условия леммы 2 , где в обоих случаях $a=2 \pi$ и

$$
\begin{aligned}
b=-\frac{1}{\rho} \operatorname{Im} \tau_{\mu}, & d=\arg c_{\mu}-\frac{\pi}{2 \rho} \operatorname{Re} \tau_{\mu}-\frac{1}{\rho}\left(\operatorname{Im} \tau_{\mu}\right) \log 2 \pi, \\
b=\frac{1}{\rho} \operatorname{Im} \tau_{\mu}, & d=-\arg c_{\mu}-\frac{\pi}{2 \rho} \operatorname{Re} \tau_{\mu}+\frac{1}{\rho}\left(\operatorname{Im} \tau_{\mu}\right) \log 2 \pi
\end{aligned}
$$

соответственно в случаях последовательностей $\left|Z_{+}\right|$и $\left|Z_{-}\right|$. При этом во втором случае следует класть $m=1$. Значит, если обозначить через $n_{ \pm}(t)$ число точек последовательности $\left|Z_{ \pm}\right|$в интервале $(0, t)$, то по лемме 2

$$
\int_{0}^{r} \frac{n_{+}(t)+n_{-}(t)}{t} d t=\frac{r^{\rho}}{\pi \rho}+\left(\frac{\operatorname{Re} \tau_{\mu}}{2 \rho}-m\right) \log r+o(\log r), \quad r \rightarrow \infty .
$$

Но $n_{+}(t)+n_{-}(t)$ есть число нулей функции $F(z)$ в круге $|z|<t$. Запишем для этой функции формулу Иенсена

$$
\int_{0}^{r} \frac{n_{+}(t)+n_{-}(t)}{t} d t=\frac{1}{2 \pi} \int_{-\pi}^{\pi} \log \left|F\left(r e^{i \theta}\right)\right| d \theta-\log |F(0)| .
$$

Сопоставляя с этой формулой оценки (35) и (21), видим, что если $\rho>1 / 2$, то $m=s$. Остается вспомнить, что $s=1$ при $\mu \neq 1 / \rho-l$ и $s=2$ при $\mu=1 / \rho-l, l \in \mathbb{Z}_{+}$. Значит, $m=1$ и $m=2$ соответственно и случай $\rho>1 / 2$ разобран.

Если $\rho=1 / 2, \operatorname{Re} \mu>3$, то вместо формулы (21) применяется формула (23). Но (23) совпадает с (21) при $\rho=1 / 2, s=1$, и случай $\rho=1 / 2, \operatorname{Re} \mu>3$ также разобран. Теорема 1 доказана.

Докажем теорему 2. По теореме А последовательность $Z$ нулей $z_{n}$ функции $F(z)$ имеет вид $Z=\left(z_{n}\right)_{n=m}^{+\infty}$, где $m$ - некоторое целое число, и

$$
\left|z_{n}\right|=\left(\frac{\pi}{\sin \pi \rho}\left(n+\frac{1}{2}+\rho(\operatorname{Re} \mu-1)+o(1)\right)\right)^{1 / \rho}, \quad n \rightarrow+\infty .
$$

Пусть $n(t)$ имеет тот же смысл, что и в лемме 2 , т.е. $n(t)$ есть число точек $z_{n}$ в круге $|z|<t$. Формула (37) показьвает, что для последовательности $|Z|$ вьполнены условия леммы $2 \mathrm{c}$

По лемме 2

$$
a=\frac{\pi}{\sin \pi \rho}, \quad b=0, \quad d=\frac{\pi}{\sin \pi \rho}\left(\rho(\operatorname{Re} \mu-1)+\frac{1}{2}\right) .
$$

$$
\int_{0}^{r} \frac{n(t)}{t} d t=\frac{\sin \pi \rho}{\pi \rho} r^{\rho}-(m+\rho(\operatorname{Re} \mu-1)) \log r+o(\log r), \quad r \rightarrow \infty .
$$

С другой стороны, в силу формулы Иенсена $(36)$, где теперь $n_{+}(t)+n_{-}(t)=n(t)$, и оценки (31) имеем

$$
\int_{0}^{r} \frac{n(t)}{t} d t=\frac{\sin \pi \rho}{\pi \rho} r^{\rho}-\rho(\operatorname{Re} \mu-1) \log r+O(1), \quad r=r_{k} \rightarrow \infty .
$$

Из (38) и (39) следует, что $m=0$. Теорема 2 доказана. 
5. Мы будем использовать следуюшие формулы:

$$
\begin{gathered}
E_{1}(z ; \mu)=\frac{1}{\Gamma(\mu-1)} \int_{0}^{1} e^{z t}(1-t)^{\mu-2} d t, \quad \mu>1, \\
(\mu-1) E_{\rho}(z ; \mu)+\frac{z}{\rho} E_{\rho}^{\prime}(z ; \mu)=E_{\rho}(z ; \mu-1), \quad \rho>0, \quad \mu \in \mathbb{C}, \\
E_{1}(z ; \mu)-E_{1}^{\prime}(z ; \mu)=(\mu-1) E_{1}(z ; \mu+1), \quad \mu \in \mathbb{C} .
\end{gathered}
$$

Формулы (40), (41) содержатся в [1, с. 120,118$]$. Формула (42) следует из определения (1) и из свойства $\Gamma(z+1)=z \Gamma(z)$. Действительно, в силу (1) левая часть в (42) есть

$$
\sum_{n=0}^{\infty}\left(\frac{1}{\Gamma(\mu+n)}-\frac{n+1}{\Gamma(\mu+n+1)}\right) z^{n}
$$

и, подставляя сюда $\Gamma(\mu+n)=\Gamma(\mu+n+1) /(\mu+n)$, мы получаем правую часть. Нам понадобится также

Теорема ПоЙа [9]. Пусть функиия $f(t)$ положительна, интегрируема и дифферениируема в интервале $(0,1)$ и пусть

$$
-\infty \leqslant \alpha \leqslant-\frac{f^{\prime}(t)}{f(t)} \leqslant \beta \leqslant+\infty, \quad t \in(0,1) .
$$

Тогда если $f(t)$ не совпадает ни с функиией $c \cdot \exp (-\alpha t)$, ни с функиией $c \cdot \exp (-\beta t)$, то все нули челой функиии

$$
F(z)=\int_{0}^{1} e^{z t} f(t) d t
$$

лежат в полосе $\alpha<\operatorname{Re} z<\beta$.

В статье [5] доказано, что при $\mu \in(0,1) \cup(1,2)$ все нули функции $E_{1}(z ; \mu)$ лежат в полуплоскости $\operatorname{Re} z<0$. Справедлива

Теорема 3. 1) При $\mu \in(1,2)$ все нули функиии $E_{1}(z ; \mu)$ лежат в полуплоскости $\operatorname{Re} z<\mu-2$.

2) При $\mu>2$ все нули функиии $E_{1}(z ; \mu)$ лежат в полуплоскости $\operatorname{Re} z>\mu-2$.

3) При $\mu \in(1,3]$ все нули функиии $E_{1}(z ; \mu)$ простьи.

ДокаЗАТЕЛЬСтво. В силу (40) функция $E_{1}(z ; \mu)$ имеет вид (43), где

$$
f(t)=\frac{(1-t)^{\mu-2}}{\Gamma(\mu-1)} .
$$

Имеем

$$
-\frac{f^{\prime}(t)}{f(t)}=\frac{\mu-2}{1-t}, \quad 0<t<1
$$


Отсюда $-f^{\prime}(t) / f(t) \leqslant \mu-2$ при $1<\mu<2$ и $-f^{\prime}(t) / f(t) \geqslant \mu-2$ при $\mu>2$, и утверждения 1), 2) следуют из теоремы Пойа.

Остается доказать утверждение 3$)$. При $\mu=2$ явньй вид $E_{1}(z ; 2)=\left(e^{z}-1\right) / z$ показывает, что все нули функции $E_{1}(z ; 2)$ просты и лежат на мнимой оси.

Пусть $1<\mu<2$. По утверждению 1$)$ все нули функции $E_{1}(z ; \mu)$ лежат в полуплоскости $\operatorname{Re} z<\mu-2<0$. Если бы у $E_{1}(z ; \mu)$ был кратный корень $z_{0}$, то по формуле $(42)$ он был бы корнем функции $E_{1}(z ; \mu+1)$ и по утверждению 2$) \operatorname{Re} z_{0}>\mu-1>0$. Противоречие.

Пусть $2<\mu \leqslant 3$. По утверждению 2$)$ все нули $E_{1}(z ; \mu)$ лежат в полуплоскости $\operatorname{Re} z>\mu-2>0$. Если бы у $E_{1}(z ; \mu)$ был кратньй корень $z_{0}$, то по формуле $(41)$ он был бы корнем функции $E_{1}(z ; \mu-1)$. По утверждению 1$)$ и в силу сказанного о нулях функции $E_{1}(z ; 2)$ мы имели бы противоречивое неравенство $\operatorname{Re} z_{0} \leqslant 0$. Теорема 3 доказана.

Рассмотрим неполную гамма-функиию

$$
\gamma(\alpha, z)=\int_{0}^{z} e^{-t} t^{\alpha-1} d t, \quad \operatorname{Re} \alpha>0
$$

переменной $z$. Эта функция аналитична всюду, за возможным исключением точки ветвления $z=0$; главная ветвь определяется с помощью разреза по отрицательной вещественной полуоси. После разложения $e^{-t}$ в степенной ряд и почленного интегрирования получаем представление

$$
\gamma(\alpha, z)=z^{\alpha} \sum_{n=0}^{\infty}(-1)^{n} \frac{z^{n}}{n !(n+\alpha)}
$$

позволяющее распространить $\gamma(\alpha, z)$ на все значения $\alpha \neq 0,-1,-2, \ldots$ Отсюда следует, что модифицированная функция $\gamma^{*}(\alpha, z)=\gamma(\alpha, z) /\left(z^{\alpha} \Gamma(\alpha)\right)$ является целой (в том числе и по $\alpha)$ и справедлива формула

$$
\gamma^{*}(\alpha, z):=\frac{\gamma(\alpha, z)}{z^{\alpha} \Gamma(\alpha)}=e^{-z} \sum_{n=0}^{\infty} \frac{z^{n}}{\Gamma(\alpha+n+1)}
$$

$[10$, c. 65$]$. Значит, в силу (1) при всех $\alpha \in \mathbb{C}$

$$
\gamma^{*}(\alpha, z)=e^{-z} E_{1}(z ; 1+\alpha) .
$$

Исключим из рассмотрения вырожденный случай $\alpha=0,-1,-2, \ldots$, в котором $\gamma^{*}(\alpha, z)=z^{-\alpha}$.

СлЕДСТВИЕ 1. Пусть $\alpha \neq 0,-1,-2, \ldots$. Тогда все нули $w_{n}$ функиии $\gamma^{*}(\alpha, w)$ мохсно пронумеровать так, что $n \in \mathbb{Z} \backslash\{0\} u$

$$
w_{n}=2 \pi n i-(1-\alpha)\left(\log 2 \pi|n|+i \frac{\pi}{2} \operatorname{sign} n\right)+\log \frac{1}{\Gamma(\alpha)}+O\left(\frac{\log |n|}{|n|}\right), \quad n \rightarrow \pm \infty
$$

При этом все нули, начиная с некоторого, просты.

Благодаря формуле (45) следствие 1 вытекает из утверждения 1) теорем А и 1 и из замечания относительно убьвания величины $o(1)$, сделанного после формулировки теоремы А.

В свою очередь, из (45) и из теоремы 3 сразу вытекает 
СлЕДСТВИЕ 2. 1) При $0<\alpha<1$ все нули функиии $\gamma^{*}(\alpha, w)$ лежсат в полуплоскости $\operatorname{Re} w<\alpha-1$.

2) При $\alpha>1$ все нули функиии $\gamma^{*}(\alpha, w)$ лежат в полуплоскости $\operatorname{Re} w>\alpha-1$.

3) При $0<\alpha \leqslant 2$ все нули функиии $\gamma^{*}(\alpha, w)$ просты.

В заключение рассмотрим функиию ошибок

$$
\operatorname{erf} z=\frac{2}{\sqrt{\pi}} \int_{0}^{z} e^{-t^{2}} d t
$$

Это целая функция. Она имеет простой корень $z=0$ и не имеет других вещественных корней. Из (44) и (47) вытекает формула

$$
\operatorname{erf} z=\frac{1}{\sqrt{\pi}} \gamma\left(\frac{1}{2}, z^{2}\right)
$$

(см. также [10, с. 65]), показывающая вместе с определением $\gamma^{*}(\alpha, z)$, что все ненулевые корни функции erf $z$ совпадают с корнями функции $\gamma^{*}\left(1 / 2, z^{2}\right)$. По следствию 2 корни функции $\gamma^{*}\left(1 / 2, z^{2}\right)$ лежат на множестве $\operatorname{Re} z^{2}<-1 / 2$, состоящем из двух криволинейных секторов, симметричных друг другу относительно вещественной оси и ограниченных компонентами гиперболы $y^{2}-x^{2}=1 / 2(z=x+i y)$. Это множество лежит в углах $| \pm \pi / 2-\arg z|<\pi / 4$. Рассмотрим отображение $w=z^{2}$ угла $|\pi / 2-\arg z|<\pi / 4$ на полуплоскость $\operatorname{Re} w<0$, в которой по следствию 2 лежат корни $w_{n}$ функции $\gamma^{*}(1 / 2, w)$, являюшиеся простыми. Обозначим через $z_{n}^{+}$корни $\operatorname{erf} z$ в угле $|\pi / 2-\arg z|<\pi / 4$. Тогда $n \in \mathbb{Z} \backslash\{0\}$ и в силу (46) с $\alpha=1 / 2$ имеем

$$
\left(z_{n}^{+}\right)^{2}=2 \pi n i-\frac{1}{2} \log |n|-i \frac{\pi}{4} \operatorname{sign} n-\log \left(\Gamma\left(\frac{1}{2}\right) \sqrt{2 \pi}\right)+O\left(\frac{\log |n|}{|n|}\right), \quad n \rightarrow \pm \infty .
$$

В силу нечетности erf $z$ в угле $|\pi / 2+\arg z|<\pi / 4$ располагается симметричная цепь $z_{n}^{-}$ корней erf $z$. Других корней у erf $z$, кроме точки $z=0$, нет. Так как $\Gamma(1 / 2)=\sqrt{\pi}$, мы доказали

СлЕдСтВИЕ 3. Все корни функции erf z просты. Ненулевые корни образуют две иепи $z_{n}^{+}$и $z_{n}^{-}, n \in \mathbb{Z} \backslash\{0\}$, симметричные друг другу относительно вещественной оси и расположенные в гиперболических секторах $y^{2}-x^{2}>1 / 2$. При этом

$$
\left(z_{n}^{ \pm}\right)^{2}=2 \pi n i-\frac{1}{2} \log |n|-i \frac{\pi}{4} \operatorname{sign} n-\log (\pi \sqrt{2})+O\left(\frac{\log |n|}{|n|}\right), \quad n \rightarrow \pm \infty .
$$

По поводу нулей неполной гамма-функции справочник Ю. Люка [11] отсылает к статье К. Кельбига [12]. В [12], равно как и в цитируемых там предшествуюших работах, представлены другие, в основном, вычислительные аспекты вопроса. 


\section{СПИСОК ЦИТИРОВАННОЙ ЛИТЕРАТУРЫ}

[1] Джрбашян М. М. Интегральные преобразования и представления функций в комплексной области. М.: Наука, 1966.

[2] Wiman A. Über die Nullstellen der Funktionen $E_{\alpha}(x) / /$ Acta Math. 1905. V. 29. P. 217-234.

[3] Polya G. Bemerkung über die Mittag-Lefflerschen Funktionen $E_{\alpha}(z) / /$ Tôhoku Math. J. 1921. V. 19. P. 241-248.

[4] Седлецкий А. М. Асимптотические формулы для нулей функции типа Миттаг-Леффлера // Anal. Math. 1994. V. 20. P. 117-132.

[5] Ostrovskiǐ I. V., Peresyolkova I. N. Nonasymptotic results on distribution of zeros of the function $E_{\rho}(z ; \mu) / /$ Anal. Math. 1997. V. 23. P. 283-296.

[6] Леонтьев А. Ф. О представлении аналитических функций в замкнутой вьпуклой области рядами Дирихле // Изв. АН СССР. Сер. матем. 1973. Т. 37. №3. С. 577-592.

[7] Леонтьев А. Ф. Ряды экспонент. М.: Наука, 1976.

[8] Седлецкий А. М. Базисы из экспонент в пространствах $E^{p}$ на выпуклых многоугольниках // Изв. АН СССР. Сер. матем. 1978. Т. 42. № 5. С. 1101-1119.

[9] Polya G. Über die Nullstellen gewisser ganzer Funktionen // Math. Z. 1918. V. 2. P. 352-383.

[10] Олвер $\Phi$. Введение в асимптотические методы и специальные функции. М.: Наука, 1978.

[11] Люк Ю. Специальные математические функции и их аппроксимации. М.: Мир, 1980.

[12] Kölbig K.S. On the zeros of the incomplete gamma function // Math. Comp. 1972. V. 26. P. 751-756.

Московский государственный университет им. М. В. Ломоносова

Поступило

14.09.1999 